\author{
LPG Gas Leakage Detection Using IOT \\ Arun Manhas ${ }^{1}$, Neeraj Chambyal ${ }^{1}$, Manish Raina ${ }^{1}$, Dr.Simmi Dutta ${ }^{2}$, Er. Prabhjot Singh ${ }^{3}$ \\ ${ }^{1}$ Department of Computer Engineering, GCET , Chak Bhalwal, Jammu , J\&K, India \\ ${ }^{2} \mathrm{HOD}$, Department of Computer Engineering, GCET , Chak Bhalwal , Jammu , J\&K, India \\ ${ }^{3}$ Assistant Professor, Department of Computer Engineering, GCET, Chak Bhalwal, Jammu, J\&K, India
}

\title{
ABSTRACT
}

Article Info

Volume 7, Issue 4

Page Number : 520-526

\section{Publication Issue :}

July-August-2021

\section{Article History}

Accepted : 07 Aug 2021

Published : 14 Aug 2021
Safety plays a major role in today's world and it is necessary that good safety systems are to be implemented in places of education and work. This work modifies the existing safety model installed in industries and this system also be used in homes and business premises. One of the preventive measures to avoid the danger associated with gas leakage is to install a gas leakage detector at vulnerable locations.

A gas detector is a device that detects the presence of gases in an area, often as part of a safety system. Gas Detector where it can sound an alarm to operators in the area where the leak is occurring, giving them the opportunity to fix or leave. This type of device is important because there are many gases that can be harmful to organic life, such as humans or animals.

This paper provides a brand new approach to discover LPG discharge supported microcontroller based Node MCU. To alert on Liquefied rock oil Gas (LPG) leakage and preventing any unwanted incident, we need to apply some cautions to discover the discharge. This paper aims to provide a solution to this problem by building a device which will do the area monitoring continuously. The gas sensor provides data to Node MCU, and then the results are displayed as a warning to the user via an Android-based smart-phone device. Other than LPG gas, Air conditioner and refrigerator leaked gases are also harmful in home. Using this device users will be able to prevent accidents that occur due to harmful gas leaks so that accidents can be avoided.

Keywords : IOT, Node MCU, LPG, Sensors

\section{INTRODUCTION}

LPG is the abbreviation or short kind for liquefied oil gas. Like all fossil fuels, it's a non-renewable supply of energy. It is extracted from fossil oil and gas. LPG gas is largely gas and alkane and it's scentless in its state of nature. The smell that we tend to notice once there's a run is really of a wholly totally different agent, referred to as alkyl radical Mercaptan. This substance is additional to the gas once it leaves the 
most storage terminals[1]. Humans cannot detect the presence of natural gases as fast as the sensor does. Thus, the use of gas sensing system is hugely needed to give real-time monitoring of the gas system.

In certain cases, gas leakage can cause fire that will destroy human property. The large scale of fire also could contribute to serious injury or death. This is due to the firestation getting delayed information about the occurrence of fire .The current products that are in the market function as a gas detector. It will only detect a gas and trigger an alarm. The main problem is that even the alarm is triggered but if the user is not at home or premise, the user will not get to know about the leakage of gas.

The prime aim of paper is to detect Gas leakage in home, hotels, schools and other domestic areas, and gives alert message to the surrounding people. Nowadays Gas sensors are being used globally in the field like safety, health, instrumentation etc. This paper is an implementation of the same using MQ-5 gas sensor. The MQ5 sensor is commonly used for detecting gas leakage for various applications. The MQ-5 gas sensor detects the concentration of gas in ppm and outputs analog value which can be converted to a digital signal. The paper allows the user to set the low, medium and dangerous level for leakage based on the same digital measure. The intensity values are compared with two predetermined thresholds and based on that, it classifies it into three different classes of concentration of leakage[2].Besides that, it is more efficient information wise as it is also capable to send out an SMS to the owner.

\section{LITERATURE SURVEY}

In the proposed system we have designed "IOT based Smart Gas Monitoring System". This proposed system aims to detect the economic fuels like petroleum, liquid petroleum gas, alcohol etc and allows a provision for controlling the gas leakage by closing the valve automatically. The next feature of the topic is to ensure the booking of gas cylinder from gas agency. The sensors detect the leaked gas and send the signal to the internet ; thereafter the programme on the internet, directs the android app using the signal for switching off gas valve from a distant place. So it redirects again to the internet and closes the gas cylinder valve through IOT. The problem of gas wastage could also be avoided using this system. Sometimes if the burner is left on by mistake, the consumer could be alerted about the problem. If the burner is on and there is no vessel on top of it, an alarm goes off.

\section{SYSTEM ARCHITECTURE}

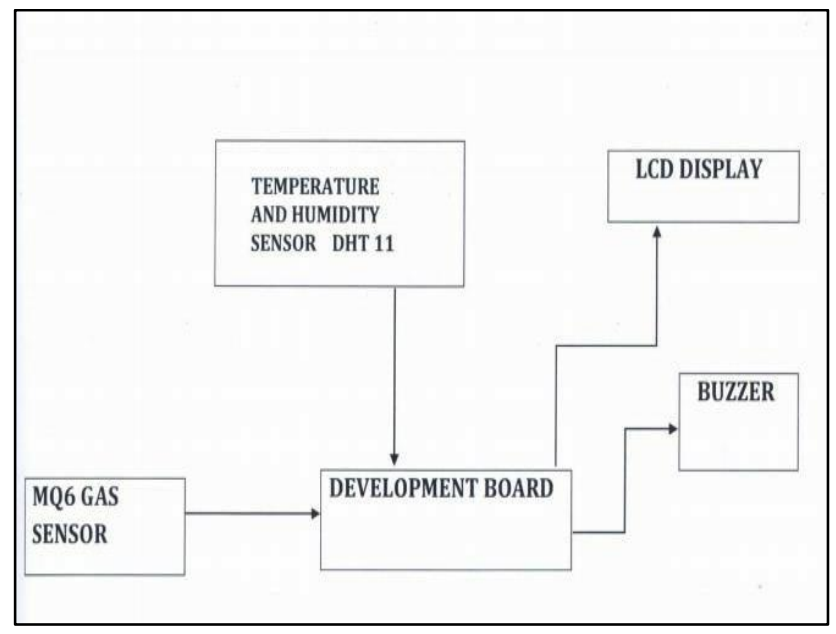

Figure-1. System Architecture

Humans cannot detect the presence of natural gases as fast as the sensor does. Thus, the gas sensing mechanism is hugely needed to give real-time monitoring of the gas system. As we know its leak may lead to a disaster. Here we have developed associate NodeMCU primarily based LPG gas detector alarm. If gas outpouring happens, this technique detects it, raises an alert by droning the buzzer connected with the circuit. This technique is simple to create and anyone who has some information of physics and programming, will build it[3]. 
Components of System Architecture

1. NodeMCU

2. LPG Gas sensor Module

3. Connecting wires

4. Jumper Wires

5. Buzzer

6. Connecting wires

\section{NodeMCU}

NodeMCU is an open source IoT platform. It includes firmware which runs on the ESP8266 WiFi SoC from Espressif Systems, and hardware which is based on the ESP-12 module. The term "NodeMCU" by default refers to the firmware rather than the development kits. The firmware uses the Lua scripting language.

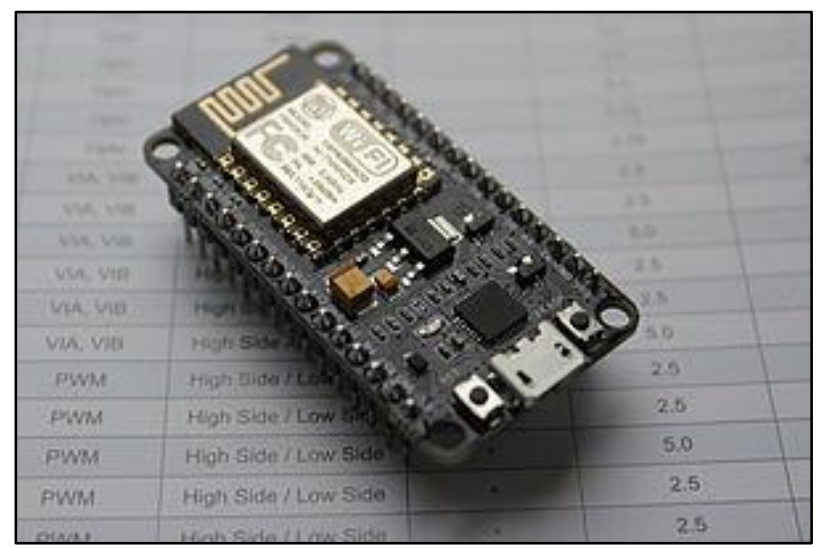

Figure 2: NodeMCUNode

Image Source:wikipedia

\section{MQ5 GAS SENSOR}

In the Youtube, experts have explained about different components of LPG architecture. The sensing element is capable of detecting Six differing kinds of combustible gases on label sensitivity. This sensing element may be label mistreatment the potentiometer fitted within the jailbreak board of MQ5[4] gas sensing element. The sensing element provides associate degree analog output. The MQ-5 will observe gas concentrations in any place from two hundred to $10000 \mathrm{ppm}$. The sensor's output is associated with the degree of analog resistance.
Interfacing with the sensing element module is finished through a 4-pin board compatible Slp header and it needs one i/o pin from the host microcontroller. The onboard microcontroller gives initial heating interval once power-up and starts to give live LPG sensing element output.

It consists of four pins, the details of LPG Gas sensor is shown in Table-1.

Table-1. Pin Specification of MQ-5 Gas sensor

\begin{tabular}{|l|l|l|l|}
\hline Pin No & Pin Name & I/0 & \multicolumn{1}{|c|}{ Details } \\
\hline 1 & VCC & Power IN & Positive power supply, 5v Regulated Power \\
\hline 2 & GND & Power GND & Ground \\
\hline 3 & ANALOG & $0 / P$ & Analog output voltage \\
\hline 4 & OUT & $0 / P$ & Output voltage \\
\hline
\end{tabular}

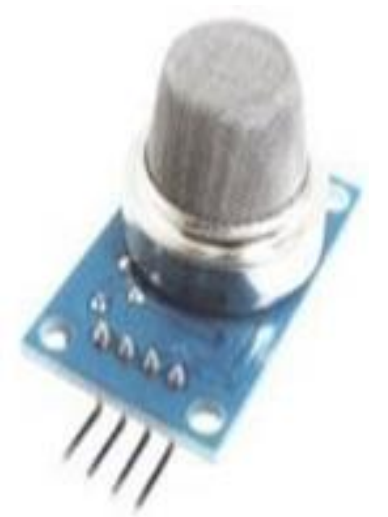

Figure 3 : MQ-5 Gas Sensor

Image Source: IndiaMART

\section{CONNECTING WIRES}

A wire may be a single, typically cylindrical, versatile strand or rod of metal. Wires square measure accustomed bear mechanical masses or electricity and telecommunications signals. Wire is often fashioned by drawing the metal through a hole in a very die or draw plate. Wire gauges are available in numerous customary sizes, as expressed in terms of a gauge range. The term wire is additionally used additional loosely to confer with a bundle of such strands, as in "multi stranded wire", that is additional properly termed a wire rope in mechanics, or a cable in electricity. Connecting wires will be made up of $\mathrm{Al}$ 
and different materials the majority electrical wires square measure fabricated from copper.

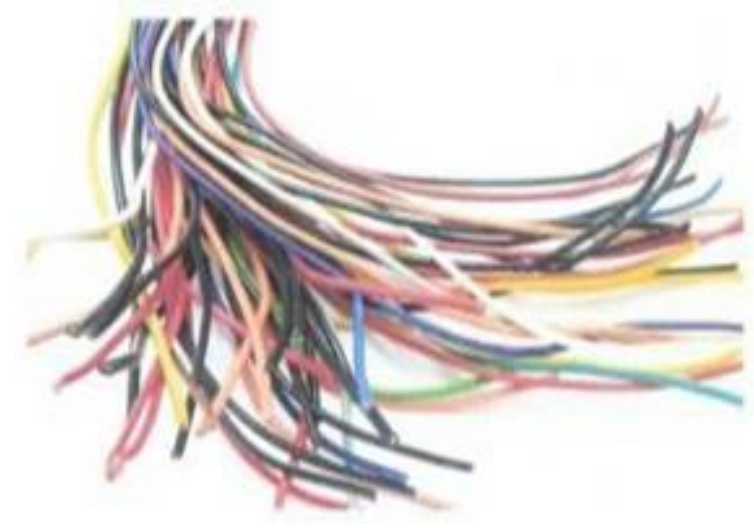

Figure 4. To depict the connecting wires

\section{JUMPER WIRES}

A jumper wire is associate electrical wire, or cluster of them forming a cable, with a connecter or pin at every finish (or typically while not them - merely "tinned"), that is often accustomed interconnect the components of a breadboard or other prototype or test circuit, internally or with other equipment or components, without soldering. Individual jump wires are fitted by inserting their "end connectors" into the slots provided during a board, the header connecter of a printed circuit, or a chunk of equipment $[5,6]$

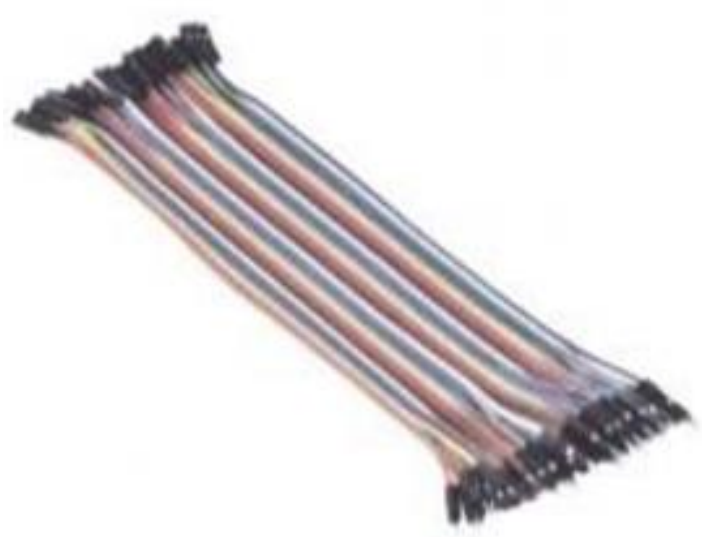

Figure-5. Jumper Wires Image Source: RAM Electronics

\section{BUZZER}

A buzzer or pager is an audio signaling device, which can be mechanical, mechanical device, or electricity (Piezo for short). It has 2 pins in it. It's easy construction and low worth makes it usable in varied applications like car/truck reversing indicator, computers, decision bells etc.[7] Once subjected to an alternating field of force they stretch or compress, in accordance with the frequency of the signal thereby producing sound.

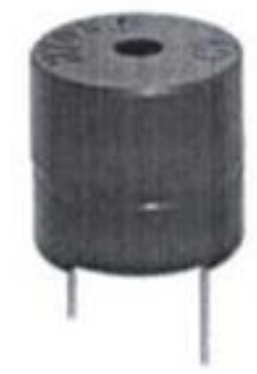

Figure-6. Buzzer

\section{PROPOSED SYSTEM}

Here we have adapted new technology IOT (Internet of Things) to get fastest notification of gas leakage. We shall use a stepper motor to OFF the knob of cylinder regulator to avoid the accidental cases due to gas leakage. We will also use a website or application under the IOT technology to get fastest response from the module. The other modules used in this project areGSM module, microcontroller, exhaust fan, LED for indication, a buzzer to notify local peoples. And MQ 5 or MQ 6 gas sensor module to sense the gas leakage. The main advantage of this work is that it can determine the leakage and send the data over to a website, where it can be monitored and corrective actions can be taken. If appropriate measures are taken quickly after it is reported over the IOT, it can help in saving the loss of life and property. 


\section{IMPLEMENTATION}

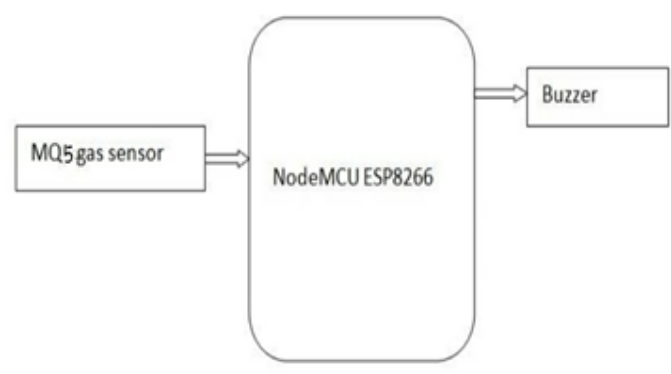

Figure-7: System Design

As shown in the diagram above (from fig-7), the system contains NodeMCU, LPG GAS device Module and buzzer. NodeMCU controls the complete method of this technique like reading LPG Gas device module output, causation message and activating buzzer.

The functioning of the circuit once the device is supercharged ON. The MQ5 device gas module has four pins. 2 pins area unit used for interfacing with development board and 2 other pins area unit VCC and ground. Out of 2 interfacing pins one pin is analog output and the other is digital pin. The analog output pin of the module is employed for detecting concentration level of gas outpouring and interfaced with the A0 analog input pin of the NodeMCU board. In this circuit, we have connected the $\mathrm{A} 0$ pin of the MQ-5 to the A0 pin of the NodeMcu module and D0 pin remains disconnected.

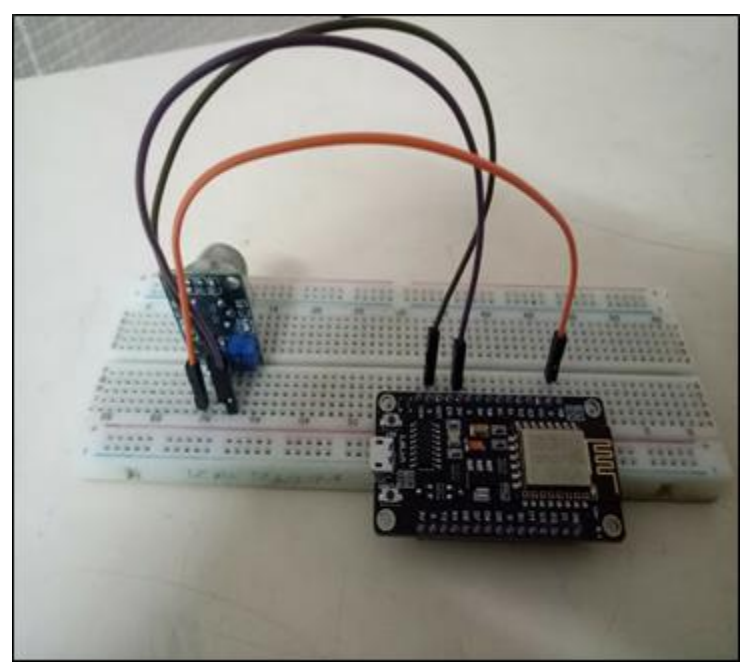

Figure-8: Circuit
Internet of Things (IoT) is the networking of 'things' by which physical things can communicate with the help of sensors, electronics, software, and connectivity. These systems do not require any human interaction and same is the case with IOT based gas detection system, it does not require human attention. IOT and Arduino based LPG leakage detection system senses the LPG gas with the help of an LPG gas sensor. LPG gas sensor interfacing with Arduino is implemented in this project. The Signal from this sensor is sent to the Arduino microcontroller. The microcontroller is connected to an LCD, Buzzer and IOT module (ESP8266). IOT LPG leakage detector project is implemented using an ESP8266 chip. This is a Wi-Fi module which is used for connecting micro-controllers to $\mathrm{Wi}-\mathrm{Fi}$ network and make TCP/IP connections and send data. Data, which is sensed by these sensors, is then sent to the IOT. The IOT module then sends the data over to a website. Once the gas leakage is detected, the buzzer is turned ON and a 'Leakage detected' message is displayed on the LCD. The Pre-requisite for this LPG gas leakage detection and the smart alerting project is that the Wi-Fi module should be connected to a WiFi zone or a hotspot. This project is also implemented without the IOT module. In place of the IOT module, we have used a GSM module, by which an SMS is triggered.[8]

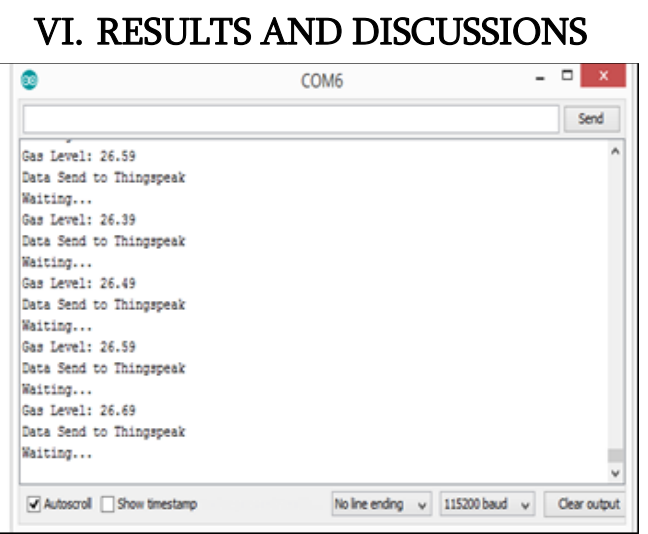

Figure-9 : Serial monitor output when gas leakage detected.

Image Source:how2electronics 


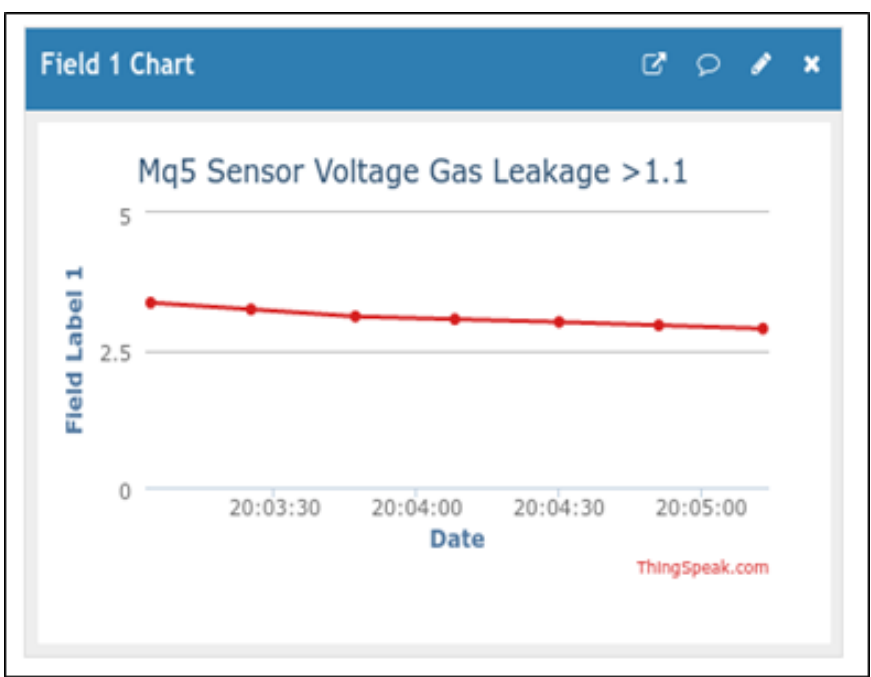

Figure-10 : Monitor Thingspeak Channel Output. Image Source:hackanons

The proposed approach can be implemented in IOT basics and stored in thinkspeaks.com. The graph representation provides the field chart for gas leakage and monitoring levels. We can monitor the sensors anywhere and anytime using cloud framework. The chart represents for gas sensor levels to identify the gas leakage. The ThinkSpeak is a Cloud in which it can receive the sensors data via Wi-Fi Module and it can represent the data as a field graph in the various parameters. The ThinkSpeak can generate the api key. The Api key can be given in source code. By Giving SSID and Password we can connect the wi-fi module with the thinkspeak services.

Here we are testing on every insertion string on channel MQ5 SENSOR VALUE, Field 1 in which the condition is - if inserted value is not equal to 0 . If this condition is true we call into the ThingHttp by setting the Action to ThingHttp. Now, whenever your IoT device sends a piece of data into ThingSpeak, ThingSpeak will automatically relay a post to PushBullet and in turn notify your devices[9].

\section{CONCLUSION AND FUTURE ENHANCEMENT}

Gas escape could result in severe accidents which ends in material losses and human injuries. Gas escape happens chiefly because of poor maintenance of apparatus and inadequate awareness of the individuals. Thus LPG escape detection is useful to stop accidents and to avoid wasting human lives. This paper presents a LPG escape detection and alert system. This technique triggers buzzer and displays the severity of the escape to alert individuals once LPG escape is detected. This technique is incredibly straightforward nevertheless reliable.

It leaves with the additional scope of improvement. Battery utilized in this technique is of $5 \mathrm{~V}$ and in future improvement a much bigger, reversible one could be used, which may sustain the gas detection module for an extended amount of time, with alert whenever battery runs out. With additional improvement like detection of Gas Concentration and changes in style the system will be more handy and price effective for the users.

\section{REFERENCES}

[1]. Vaishnavi et.al (2014) "Intelligent LPG Leakage Detection", International Journal Of Scientific \& Engineering Research, Vol. 5, Issue 11, 2014.

[2]. http://Centrallibrary.Cit.Ac.In/Dir/Project $\% 20$ Repo rt/2018/Diploma/ETE/Detection\%20of\%20lpg\% 20le akage\%20using\%20arduino.Pdf

[3]. https://Www.Scribd.Com/Document/40421328 6/M icrocontroller-Based-LPG-Leakage-Detect1-Docx.

[4]. https:/Www.Youtube.Com/Watch?V=877wiaa nit

[5]. Akarsh Agarwal et.al (2019), "R.F.L. Safety Kit for Domestic LPG", International Journal of Innovative Research in Electrical, Electronics, Instrumentation and Control Engineering, IJIREEICE, Vol. 7, Issue 5, May 2019. DOI 10.17148/IJIREEICE.2019.7514.

[6]. http://www.aut.upt.ro/ ${ }^{\sim}$ palstefan.murvay/paper $\mathrm{s} / \mathrm{s}$ 
urvey_gas_leak_detection_localization_techniq ues.pdf

[7]. https://www.flir.com/discover/instruments/gasd etection/remote-detection-and-localization-ofgasleaks-with-autonomous-mobile inspectionrobots-intechnical-facilities/

[8]. https://wenku.baidu.com/view/f8dc5e32be2348 2f b4da4c69.html

[9]. https://hackanons.com/2018/01/iot-based-lpgcng-gas-leakage-detection-alert-using-arduinouno-esp8266-thingspeak.html

\section{Cite this article as :}

Arun Manhas, Neeraj Chambyal, Manish Raina, Dr. Simmi Dutta, Er. Prabhjot Singh, "LPG Gas Leakage Detection Using IOT", International Journal of Scientific Research in Computer Science, Engineering and Information Technology (IJSRCSEIT), ISSN : 2456-3307, Volume 7 Issue 4, pp. 520-526, JulyAugust 2021. Available at doi : https://doi.org/10.32628/CSEIT2174121

Journal URL : https://ijsrcseit.com/CSEIT2174121 